\title{
Shelf to basin shuttling of iron in oxygen deficient zones
}

\author{
KENNETH M BOLSTER ${ }^{1}$, RICHARD KEIL ${ }^{2}$, JACQUELYN \\ A NEIBAUER $^{2}$ AND JAMES MOFFETT ${ }^{1}$ \\ ${ }^{1}$ University of Southern California \\ ${ }^{2}$ University of Washington \\ Presenting Author: kbolster@usc.edu
}

Elevated concentrations of dissolved iron(II) have been observed in oxygen deficient zones (ODZs). Although oxygen concentrations in these areas are negligible, nitrate and nitrite are found in high enough concentrations to make iron oxidation thermodynamically favorable. This oxidation process is believed to be a key component of the shelf to basin shuttle, which enables the transportation of significant quantities of iron away from continental margins to the open ocean. Despite the importance of this process, the rates of these reactions are poorly constrained. We have tested a stable isotope incubation approach to measure the rate of iron oxidation in anaerobic environments under trace metal clean conditions, and deployed this technique at several sites in the Eastern Tropical North Pacific ODZ. Using this dataset combined with rate estimates from other studies, we have developed an approach to predict iron oxidation rates based on other geochemical parameters, enabling more extensive modeling of the shelf to basin shuttle and the role of ODZs in the marine iron cycle. 